\title{
Slow recovery of the magnetisation after a sub-picosecond heat pulse
}

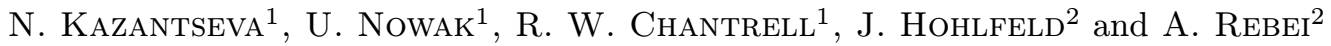 \\ ${ }^{1}$ Department of Physics, University of York - York, YO10 5DD, UK \\ ${ }^{2}$ Seagate Research - 1251 Waterfront Place, Pittsburgh, PA, 15222, USA
}

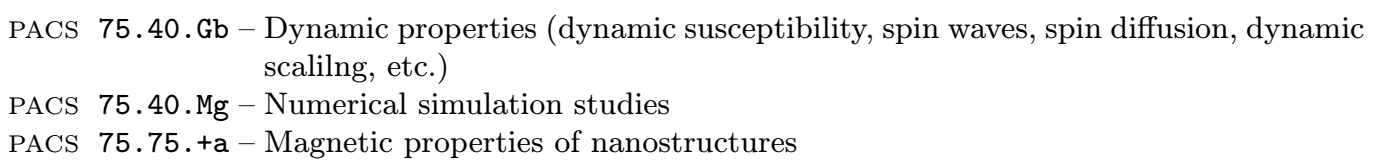

\begin{abstract}
The response of a magnetic spin system to pulsed laser heating on time scales in the picosecond regime is investigated using an atomic level classical spin Hamiltonian, the dynamics of which are based on the stochastic Landau-Lifshitz-Gilbert equation. It is found that the ferro- to paramagnetic phase transition can occur in less than one picosecond, in agreement with published experimental data. Calculating the spin temperature via the internal energy of the spin system we find that the system does not necessarily fully demagnetize even for spin temperatures above the Curie temperature. Our findings suggest that the spin system is far from thermal equilibrium so that the concept of a spin temperature has to be questioned on the time scale of picoseconds. Most importantly, the time for recovery of the magnetization can vary by orders of magnitude depending on the magnetic state after heating, a prediction which is verified by supporting pumpprobe experiments.
\end{abstract}

Laser-induced spin dynamics in the pico- and subpicosecond regime opens new perspectives for applications in magnetic storage devices, spintronics, and quantum computation. Writing information on time scales below conventional or even precessional magnetisation reversal schemes $[1,2]$ appears to be within reach. Of fundamental interest in this context is the understanding of de- and remagnetisation processes, reflecting the fundamental physical mechanisms involved in the coupling between spin, charge and lattice. Experimentally, at the forefront of these investigations is the use of pump-probe techniques. These involve the use of a high-energy femtosecond laser to cause local rapid heating of a ferromagnetic material with a consequent change of magnetisation which is measured using the magneto-optical Kerr effect. Using this technique Beaurepaire et al. [3] have shown that the magnetisation of $\mathrm{Ni}$ can be affected on the time scale of one picosecond - findings which were later confirmed by many other groups [4-8].

Optical excitations of metallic systems by ultrashort laser pulses lead to a non-equilibrium between the temperatures of the electron gas, $T_{e}$, and of the lattice, $T_{l}$, that relaxes via electron-phonon scattering. The corresponding dynamics is usually described in terms of the so-called two-temperature model that ignores any possible non-equilibrium behaviour within the electron and phonon systems but leads to very good agreement with experiment, in particular when the mean free path of initial ballistic energy transport by non-equilibrium electrons is incorporated [9]. In order to describe the electron, lattice and spin dynamics following optical excitations of metallic ferromagnets by ultrashort laser pulses, the two-temperature model has been extended to a three-temperature model [10]. Here, metallic ferromagnets are described in terms of three subsystems - electrons, phonons and magnons - with individual heat capacities, temperatures and mutual interactions.

In further analogy to the two-temperature model, the applications of the three-temperature model were based on the assumption that the mutual interactions between the subsystems can be described via temperature-independent coupling constants. Although fair reproduction of experimental data was obtained when the electron-spin interaction was assumed to be much larger than that between phonons and spins [3], the whole approach lacks microscopic justification. In particular, the assumption 
that the electron-magnon and phonon-magnon interactions could be described by temperature-independent coupling constants is more than questionable. This becomes obvious when recalling the rigorous derivation of the electron-phonon coupling constant [11] that requires both energy-independent scattering probabilities and a linear increase of the number of scatterers with the temperature of the respective subsystem. Furthermore, we will show that on a time scale of tens of picoseconds the spin system itself is not necessarily in equilibrium.

In this paper we introduce a more detailed analysis of the dynamic response of a magnetic system to pulsed laser heating in terms of an atomistic-model-based Landau-Lifshitz-Gilbert (LLG) equation with Langevin dynamics. We estimate the electron temperature from a two-temperature model calculation. Assuming that the electrons act as heat bath for the spin system, we show that Langevin dynamics is capable of reproducing the rapid decrease of the magnetisation observed in experiment. The recovery of the magnetisation on cooling is strongly dependent on the existence of some "memory" of the initial magnetic state and can be very slow when the system was fully demagnetised. Our findings demonstrate that the spin temperature is not well defined on time scales of tens of picoseconds with that questioning the value of the three-temperature model.

The magnetic system is modelled at the atomistic level, using a classical spin Hamiltonian with the Heisenberg form of the exchange interaction. Other contributions to the Hamiltonian may arise from a local, uniaxial magnetic anisotropy and the applied magnetic field, so that the Hamiltonian is given as

$$
\mathcal{H}=-\frac{J}{2} \sum_{\langle i, j\rangle} \boldsymbol{S}_{i} \cdot \boldsymbol{S}_{j}-D \sum_{i}\left(S_{i}^{z}\right)^{2}-\mu_{s} \boldsymbol{B} \cdot \sum_{i} \boldsymbol{S} .
$$

The spins $\boldsymbol{S}_{i}$ are unit vectors and we use values for the exchange integral $J$, the anisotropy constant $D$ (with the $z$-axis being the easy axis) and spin magnetic moment $\mu_{s}$ corresponding to Ni. We assume that none of these parameters changes during the heating and cooling process. This is certainly a simplification but we will show in the following that even such a simplified model gives a good qualitative description of the experiments.

Spin dynamics is modelled using the LLG equation,

$$
\frac{\partial \mathbf{S}_{i}}{\partial t}=-\frac{\gamma}{\left(1+\lambda^{2}\right) \mu_{s}} \mathbf{S}_{i} \times\left[\mathbf{H}_{i}(t)+\lambda\left(\mathbf{S}_{i} \times \mathbf{H}_{i}(t)\right)\right],
$$

where $\gamma$ is the gyromagnetic ratio and $\lambda$ a phenomenological coupling constant which governs the energy transfer between the conduction electrons and the spin system. Note, that this microscopic coupling constant is not identical to the macroscopic damping constant $\alpha$ after Gilbert, since $\alpha$ is a macroscopic parameter whose value can depend on the measurement. It has been shown for example [12], comparing an atomistic model with a macrospin model, that the macroscopic damping has a temperature dependence, even when the microscopic coupling parameter is held constant with temperature. Only at zero temperature do these two parameters coincide. The internal field $\mathbf{H}_{i}$ includes the stochastic field $\mathbf{h}_{i}$ due to the interaction of the conduction electrons with the local spins. The field $\mathbf{h}_{i}$ is assumed to be random and hence its two-point correlation functions carry memory [13]. In the following this memory will be neglected and, as usual, the random field is assumed Gaussian and satisfies

$$
\left\langle h_{i}^{a}(t) h_{j}^{b}\left(t^{\prime}\right)\right\rangle=\delta_{i j} \delta_{a b} \delta\left(t-t^{\prime}\right) 2 \lambda k_{B} T_{e} \mu_{s} / \gamma,
$$

where $a, b=x, y, z$. This guarantees the correct statistical properties in equilibrium. This approximation greatly simplifies our simulations and is enough to correctly interpret our experimental data. The resulting stochastic differential equation is solved using methods described in ref. [14].

With the assumptions above, we neglect the spinphonon coupling which might lead to additional damping mechanisms especially on time scales of a few picoseconds where electron and phonon temperature differ. Furthermore, we neglect any spational and temporal variations in the dissipation mechanism. Over all, our model is certainly not intended to contribute to the microscopic understanding of the underlying coupling mechanism between spins and conduction electrons, which might well be phononmediated [15] to enable dissipation of angular momentum. Our work does not contradict earlier theories $[15,16]$ but is focused on the thermodynamics of the spin system, simplifying the spin-electron interaction such that the conduction electrons act as heat bath for the spins.

Let us start by estimating the relevant time scales which arise in the LLG equation. During the initial rapid heating process the largest contribution in eq. (2) comes from the noise within the first cross product term leading to a minimal time scale for the demagnetisation of the order of

$$
\tau_{\mathrm{dis}} \approx \mu_{s} /\left(2 \lambda \gamma k_{B} T_{e}\right)
$$

For the model parameters of $\mathrm{Ni}$, with an $\lambda$ of 0.1 and for an electron temperature equal to the Curie temperature this simple estimate leads to a time scale of about 100 femtoseconds, in accordance with experiments and recent theoretical considerations [16]. On the other hand, during the reordering process the largest contribution in eq. (2) comes from the exchange field which, in the second term of the LLG equation, tries to align the spins towards ferromagnetic order. Assuming that there is an exchange field (at most) of the order of $z J$, where $z$ is the number of nearest neighbours, a time scale for the reordering process is at least of the order of

$$
\tau_{\mathrm{re}} \approx \mu_{s} /(\lambda \gamma z J)
$$

This time scale is of the same order of magnitude as that one for the disordering process. However, in the following 

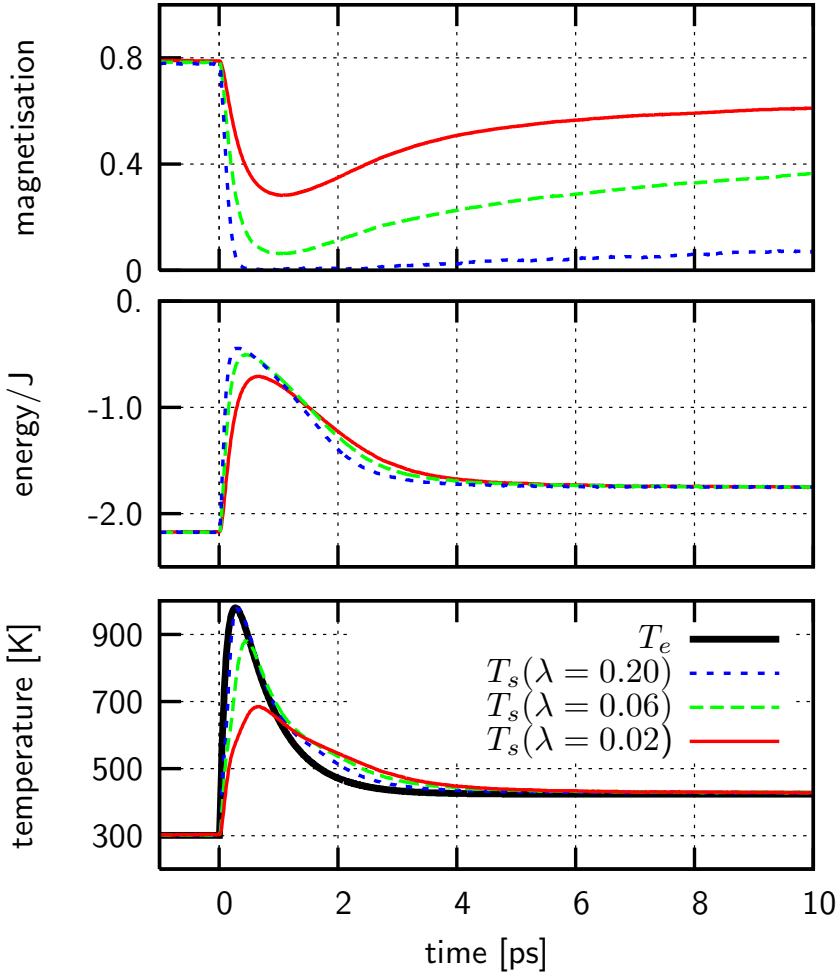

Fig. 1: (Colour on-line) Time dependence of reduced magnetisation, internal energy per spin, and spin temperature for various values of the coupling parameter $\lambda$. A stronger coupling leads to a higher-peak spin temperature and a higher degree of demagnetisation. While the recovery of the energy is faster for stronger coupling the recovery of the magnetisation is much slower.

we will show that due to the many spin degrees of freedom the reordering process is rather complex and determined by the degree of demagnetisation rather than simply by the coupling constant. The equation above is a lower limit, describing the fastest possible relaxation process.

We simulate cubic spin systems of size $80^{3}$ with periodic boundary conditions, starting by equilibrating the system at room temperature $(300 \mathrm{~K})$. Then we study the loss and recovery of the magnetisation for electron temperature sequences $T_{e}(t)$ calculated from a two-temperature model, closely resembling experimental data for thin nickel films [17]. The electron temperature is shown in the lower part of fig. 1 . Note, that for realistic parameters the maximum electron temperature can easily exceed the Curie temperature which is about $600 \mathrm{~K}$ for Ni. On a time scale of less than $5 \mathrm{ps}$ electron and lattice temperature equilibrate.

The corresponding magnetisation data of fig. 1 demonstrate significant phenomena, at short and long time scales. Firstly, we note the rapid decrease of the magnetisation on a time scale of a few ps, commensurate with the duration of the heat pulse. This is consistent with experiments $[3,4]$, and indicates that the LLG equation at the atomistic length scale contains the basic physics to describe ultrafast demagnetisation. Furthermore,

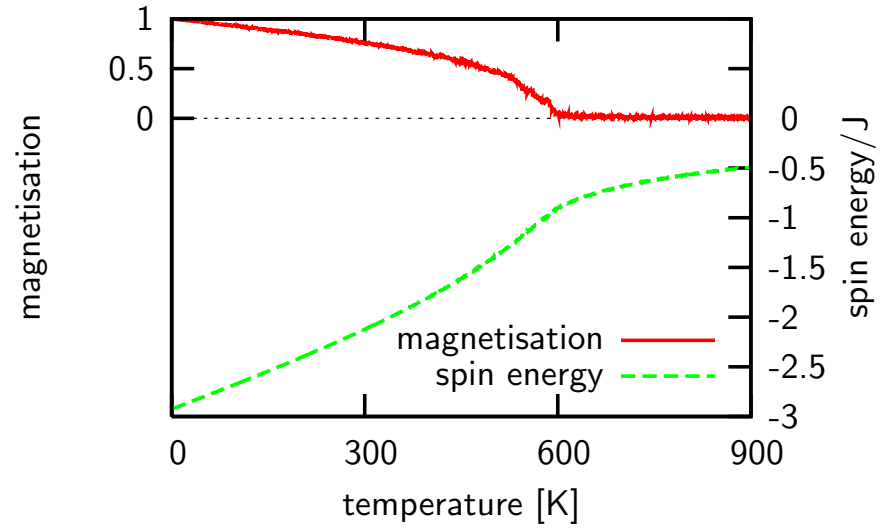

Fig. 2: (Colour on-line) Magnetisation and internal spin energy energy $v s$. temperature in equilibrium state. This energy $v s$. temperature relation is used to define an instantaneous spin temperature during our dynamics simulations.

we notice that the minimum magnetisation achieved decreases with $\lambda$. This result is explained by the presence of $\lambda$ in eq. (3), a direct consequence of the fluctuationdissipation theorem, stressing the fact that $\lambda$ is correctly considered as the parameter determining the rate at which energy can be transferred either into or out of the spin system.

Another important observation from fig. 1 is the surprisingly slow recovery of the magnetisation after the heat pulse for large values of $\lambda$ where the system is fully demagnetised. For a deeper understanding of this effect we calculate the internal energy of the spin system. The results are also shown in fig. 1. In equilibrium spin, electron and lattice temperature are equal and the temperature can be expressed as a unique function of the energy, $T(E)$ (not of the magnetisation, which is zero above $T_{c}$ ) Hence, from a separate equilibrium calculation of the temperature dependent energy (see fig. 2) we are able to estimate an effective spin temperature $T_{s}$ : at any time during the heating and subsequent cooling process the energy of the system is calculated and by comparison with the equilibrium $T(E)$ curve we can define a spin temperature. These data are also shown in fig. 1 along with the electron temperature, for comparison.

One surprising result is that the spin temperature calculated from the energy exceeds the Curie temperature in all cases, while the magnetisation curves would suggest that only in the strong coupling case $(\lambda=0.2)$ the system was totally demagnetised. Obviously, the spin system is not in equilibrium but rather in an extraordinary non-equilibrium situation where magnetic states exist with a large exchange energy, leading to apparent spin temperatures above the Curie temperature, but still with some memory of the initial magnetic state.

The other surprising result in fig. 1 is that the recovery of the magnetisation after the heat pulse can be much slower than the recovery of the internal energy (or spin temperature). An explanation for this lies in the highly 


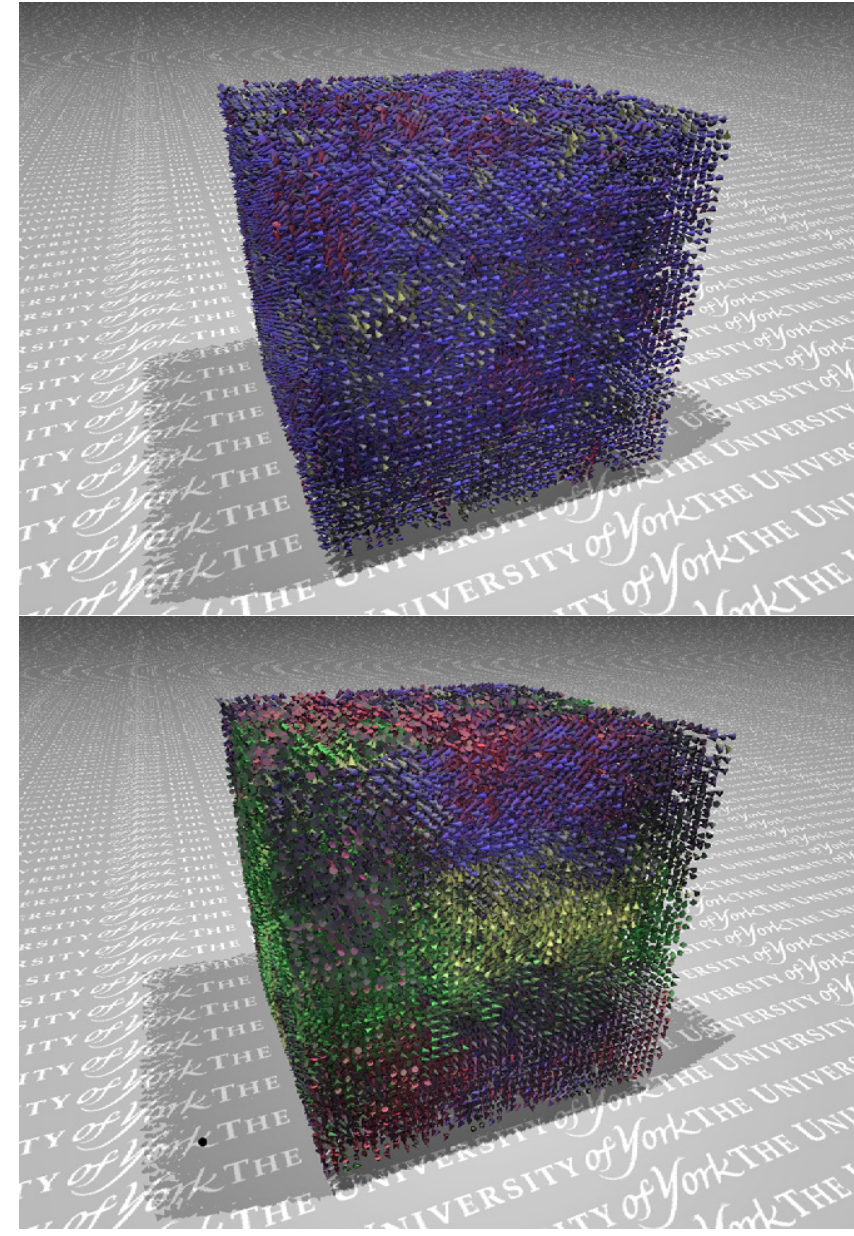

Fig. 3: (Colour on-line) Snapshots of the magnetisation distribution after $19 \mathrm{ps}$ for $\lambda=0.02$ (above, lambda02. avi ca. $2.5 \mathrm{Mb}$ ) and $\lambda=0.2$ (below, lambda20.avi ca. $2.5 \mathrm{Mb}$ ). The colours indicate the different directions of the magnetisation.

disordered state produced on complete demagnetisation. The recovery of the magnetisation from such a state will involve the growth of many small magnetically ordered regions of the order of the magnetic correlation length. After full demagnetisation the direction of the magnetisation of these initial ordered regions is random, leading to considerable frustration and a slowing-down of the recovery of the magnetisation.

To illustrate this, fig. 3 shows snapshots of the magnetisation distribution after $19 \mathrm{ps}$ for $\lambda=0.02$ and $\lambda=0.2$. While the first one is already completely ordered, the second one shows only local order but with different local magnetisation orientations, so that globally the magnetisation remains zero. Note, that this magnetisation distribution is not an equilibrium domain state but still a dynamic non-equilibrium state. For a Ni system of about $24 \mathrm{~nm}$ in lateral size the ground state is certainly single domain. Obviously, such a magnetisation distribution can only appear in cases where during the heat pulse the memory of the original magnetisation direction was completely lost, i.e., when the system was totally demagnetised.

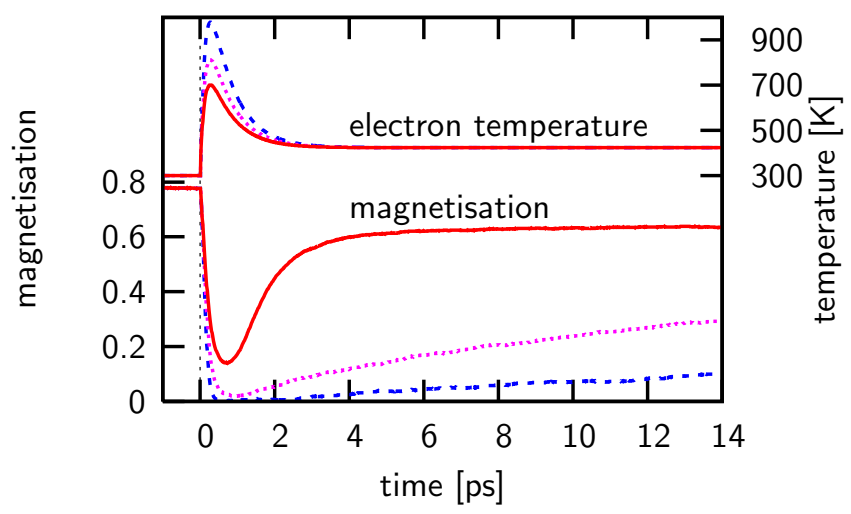

Fig. 4: (Colour on-line) Dynamics of the reduced magnetisation for different peak electron temperatures. Higher-peak electron temperatures lead to a higher degree of demagnetisation and a slower recovery.

To investigate this further we control the demagnetisation of the system in fig. 4 by varying the peak electron temperature but keeping the coupling parameter constant. Once again, slow recovery of the magnetisation is associated with a disappearance of the magnetisation. These findings strongly suggest that the recovery of the magnetisation is crucially dependent on the magnetic state achieved after the laser pulse. The dependence on $\lambda$ apparent in fig. 1 is simply a consequence of the role played by $\lambda$ in transferring energy from the conduction electrons into the spin system.

The realisation of completely demagnetised states in femtosecond pump-probe experiments is a very difficult task. Laser-pulse-induced damage and/or sample modification as well as insufficient ratios of pump and probe spot sizes are two of the most prominent reasons that hinder a thorough study of the magnetisation recovery from demagnetised states. Consequently, there are only a few results reported in the literature [18-21] and the reported transients are not conclusive. According to our model predictions the experimental difficulties to demagnetise bulk-like $\mathrm{Ni}$, Co and permalloy most probably reflect the fact that their small values of $\lambda$ hinder an effective heating of the spin system. Our results also indicate, that the slow recovery of the magnetisation does not depend on the way the demagnetised state was reached and that longer heat pulses might demagnetise a sample more efficiently than short ones.

The rapid disappearance of the magnetisation has been demonstrated experimentally on a number of occasions, and the aim here is to investigate the theoretical prediction of the slow recovery of the magnetisation from the demagnetised state. The completely demagnetised state is difficult to obtain with short, high energy, laser pulses due to film damage. Thus, we decided to use lower-power pulses of $10 \mathrm{ps}$ duration to study the breakdown and recovery of the magnetization for different excitation densities via the linear optical Kerr effect. The investigated sample consists of a glass substrate, metallic seed and adhesion layers of 


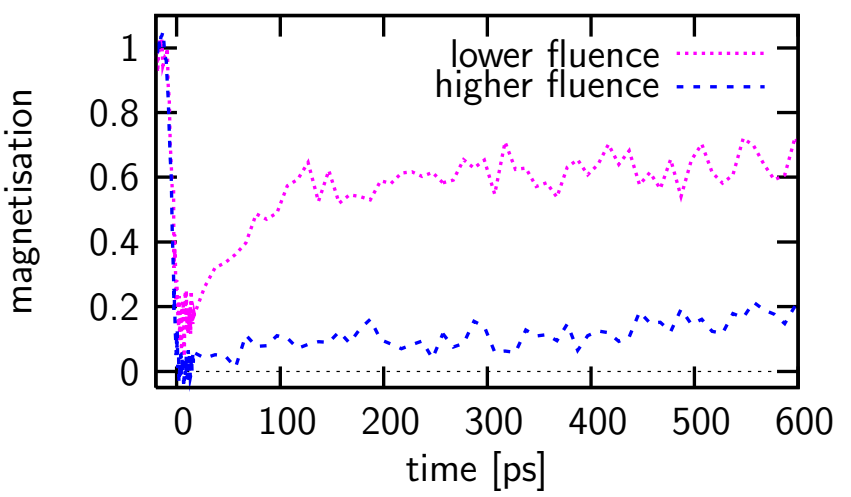

Fig. 5: (Colour on-line) Dynamics of the reduced magnetisation measured in a $\mathrm{Co} / \mathrm{Pt}$-based media for different laser fluences. A higher fluence leads to a higher degree of demagnetisation and a slower recovery.

$34 \mathrm{~nm}$ total thickness, and a perpendicular magnetized $18 \mathrm{~nm}$ thick $\mathrm{Co} / \mathrm{Pt}$ multilayer with a coercive strength of $25 \mathrm{kG}$ and a Curie temperature of $650 \mathrm{~K}$. Using pump and probe wavelength of $800 \mathrm{~nm}$ and $400 \mathrm{~nm}$, respectively, we used spectral filtering to enable the detection of the Kerr signal due to the weak probe-pulses without any interference of the strong pump-pulses (both beams hit the sample at normal incidence and a small fraction of the reflected light is used for Kerr detection). The ratios of the pump and probe energies and of the pump and probe spot sizes were approximately $100: 1$ and $20: 1$, respectively. Since the spot sizes were only estimated from visual inspection through a microscope with a $10 \mu \mathrm{m}$ scale we cannot give exact values for the pump fluence but only the energy of the pump-pulses used.

The experimental results obtained for two different pump-pulse energies of 90 and $110 \mu \mathrm{J}$, shown in fig. 5, are in excellent qualitative agreement with our predictions. The data for the weaker excitation that leads only to partial demagnetisation $\left(M_{\min } \approx 15 \%\right)$ show two distinct regimes for the magnetisation recovery. Initially, a relatively fast recovery of the magnetisation is observed during the first $100 \mathrm{ps}$ that is followed by a slow recovery at longer delays. The first regime reflects the magnetic response to fast cooling due to heat diffusion within the metallic seed layers, while further recovery during the second regime is restricted by the slow cooling due to heat diffusion into the glass substrate. In contrast, the magnetisation recovery from the completely demagnetised state created by the strong excitation is very slow at all times as predicted by our model. We note that the slow recovery of the magnetisation appears only when the completely demagnetised state is achieved. In addition, the experimental time scale for recovery is even longer than those in fig. 1. However, we do find long recovery times (of the order of ns) in calculations starting from a completely random state, as will be published elsewhere. This suggests that the low-power long-duration laser pulses are effective in achieving complete demagnetisation on a time scale of $10 \mathrm{ps}$. These findings provide experimental support for our theoretical approach.

In conclusion, we find that the experimental observations of a sub-picosecond time scale for laser-induced demagnetisation of a ferromagnet is in excellent agreement with a thermal magneto-dynamic description via the stochastic LLG equation on an atomistic level. The fact that we simulate the spin system with its microscopic thermodynamic degrees of freedom leads to a deeper understanding of the ordering dynamics, including several rather surprising facts. Firstly, even for spin temperatures above the Curie temperature the system does not necessarily fully demagnetise during the heat pulse. We conclude that the spin system is so far from equilibrium that the concept of a spin temperature has to be questioned on the time scale of picoseconds. Furthermore, we find the time scale of the recovery of the magnetisation to depend crucially on the degree of disorder of the magnetic state after heating. This prediction is verified by its agreement with the presented experimental results. These findings have a significant bearing on the prospects for heat-assisted magnetic writing schemes. In particular, any writing scheme involving full demagnetisation is limited by the time scale imposed by the slow recovery of the magnetisation. Our results suggest that further research on reversal under applied fields is vital to provide the understanding necessary to further develop heat-assisted writing processes.

\section{REFERENCES}

[1] Gerrits Th., van den Berg H. A. M., Hohlfeld J., Bär L. and RASing Th., Nature, 418 (2002) 509.

[2] Kimel A. V., Kirilyuk A., Usachev P. A., Pisarev R. V., BALbashov A. M. and RAsing Th., Nature, 435 (2005) 655.

[3] Beaurepaire E., Merle J.-C., Daunois A. and Bigot J. Y., Phys. Rev. Lett., 76 (1996) 4250.

[4] Hohlfeld J., Matthias E., Knorren R. and Bennemann K. H., Phys. Rev. Lett., 78 (1997) 4861.

[5] Scholl A., Baumgarten L., Jacquemin R. and Eberhardt W., Phys. Rev. Lett., 79 (1997) 5146.

[6] Aeschlimann M., Bauer M., Pawlik S., Weber W., Burgermeister R., Oberli D. and Siegmann H. C., Phys. Rev. Lett., 79 (1997) 5158.

[7] Koopmans B., van Kampen M., Kohlhepp J. T. and De Jonge W. J. M., Phys. Rev. Lett., 85 (2000) 844.

[8] Regensburger H., Vollmer R. and Kirschner J., Phys. Rev. B, 61 (2000) 14716.

[9] Hohlfeld J., Wellershoff S.-S., Guedde J., Conrad U., Jaehnke V. and Matthias E., Chem. Phys., 251 (2000) 237.

[10] Zhang G., Hübner W., Beaurepaire E. and Bigot J.-Y., in Spin Dynamics in Confined Magnetic Structures I, edited by Hillebrands B. and Ounadjela K. (Springer-Verlag, Berlin, Heidelberg, New York) 2002, p. 245.

[11] Allen P. B., Phys. Rev. Lett., 59 (1987) 1460. 
[12] Chubykalo-Fesenko O., Nowak U., Chantrell R. W. and Garanin D., Phys. Rev. B, 74 (2006) 094436.

[13] Rebei A. and Simionato M., Phys. Rev. B, 71 (2005) 174415.

[14] NowaK U., in Annual Reviews of Computational Physics $I X$, edited by Stauffer D. (World Scientific, Singapore) 2001, p. 105.

[15] Koopmans B., Kicken H. H. J. E., van Kampen M. and De Jonge W. J. M., J. Magn. ES Magn. Mater., 286 (2005) 271.

[16] Koopmans B., Ruigrok J. J. M., Dalla Longa F. and De Jonge W. J. M., Phys. Rev. Lett., 95 (2005) 267207.
[17] Rhie H.-S., Dürr H. A. and Eberhardt W., Phys. Rev. Lett., 90 (2003) 247201.

[18] Beaurepaire E., Maret M., Halté V., Merle J.-C., Daunois A. and Bigot J.-Y., Phys. Rev. B, 58 (1998) 12134.

[19] Conrad U., Güdde J., JÄhnKe V. and Matthias E., Appl. Phys. B: Laser Opt., 68 (1999) 511.

[20] Güdde J., Conrad U., Jähnke V., Hohlfeld J. and Matthias E., Phys. Rev. B, 59 (1999) R6608.

[21] Hohlfeld J., Gerrits Th., Bilderbeek M., Rasing Th., Awano H. and Ohta N., Phys. Rev. B, 65 (2002) 012413. 